\title{
Tunable Optofluidic Third Order DFB Dye Laser
}

\author{
Gersborg-Hansen, Morten; Kristensen, Anders
}

Published in:

Proceedings of CLEO/QELS 2007

Link to article, DOI:

10.1109/CLEO.2007.4452362

Publication date:

2007

Document Version

Publisher's PDF, also known as Version of record

Link back to DTU Orbit

Citation (APA):

Gersborg-Hansen, M., \& Kristensen, A. (2007). Tunable Optofluidic Third Order DFB Dye Laser. In Proceedings of CLEO/QELS 2007 (pp. 1-2). IEEE. https://doi.org/10.1109/CLEO.2007.4452362

\section{General rights}

Copyright and moral rights for the publications made accessible in the public portal are retained by the authors and/or other copyright owners and it is a condition of accessing publications that users recognise and abide by the legal requirements associated with these rights.

- Users may download and print one copy of any publication from the public portal for the purpose of private study or research.

- You may not further distribute the material or use it for any profit-making activity or commercial gain

- You may freely distribute the URL identifying the publication in the public portal

If you believe that this document breaches copyright please contact us providing details, and we will remove access to the work immediately and investigate your claim. 


\title{
Tunable Optofluidic Third Order DFB Dye Laser
}

\author{
Morten Gersborg-Hansen and Anders Kristensen \\ MIC - Department of Micro and Nanotechnology, Nano DTU, Technical University of Denmark (DTU), \\ Building 345east, Ørsteds Plads, DK-2800 Kongens Lyngby, Denmark. \\ www.mic.dtu.dk/nil \\ mgh@mic.dtu.dk
}

\begin{abstract}
We present a low-threshold polymer-based nanofluidic dye laser. By employing a third order DFB laser resonator, we demonstrate a threshold fluence of $\sim 7 \mu \mathrm{J} / \mathrm{mm}^{2}$ and a tunability of $45 \mathrm{~nm}$ using a single laser dye. (C)2007 Optical Society of America OCIS codes: (140.2050) Dye Lasers; (140.3490) Lasers, distributed-feedback; (140.3600) Lasers, tunable
\end{abstract}

\section{Introduction}

The advancement of microfabrication techniques for lab-on-a-chip systems has enabled integration of optical and fluidic functionalities, forming the field of 'optofluidics' [1]. One subject of particular interest is to integrate tunable light sources to lab-on-a-chip microsystems [2,3]. One approach for compact integrable light sources is microfluidic dye lasers in which a liquid laser dye is pumped through a microfluidic channel with an embedded optical resonator. Distributed feedback (DFB) laser resonators have proven particularly suitable for obtaining single mode and low threshold laser light emitted in the chip plane $[4,5,6]$.

Here, we present the design and operation of a low-threshold widely tunable polymer-based nanofluidic DFB dye laser. The device relies on light-confinement in a nanostructured polymer film where an array of nanofluidic channels constitutes a third order Bragg grating DFB laser resonator with a central $\pi / 2$ phase shift. The low reflection order of the Bragg grating yields low out-of-plane scattering loses and the sub-wavelength dimensions of the resonator segments yield a low coupling loss for the light when traversing the dye-filled nanofluidic channels, enabling a low threshold for lasing. The large free spectral range $(F S R)$ of the third order Bragg reflection of the grating facilitates wavelength tuning over the full gain spectrum of the chosen laser dye. We demonstrate laser threshold fluences down to $\sim 7 \mathrm{~J} / \mathrm{mm}^{2}$ and a wavelength tunability of $45 \mathrm{~nm}$ using a single laser dye.

\section{Third order optofluidic DFB dye laser}

The laser resonator structure, see Fig. 1, is based on a planar polymer waveguide structure supporting a single propagating TE-TM mode. The basic waveguide structure consists of a $\mathrm{SiO}_{2}$ buffer substrate $(n=1.46)$, a $300 \mathrm{~nm}$ thick core of the negative-tone resist SU-8 $(n=1.59)$, and a top cladding of poly-methylmethacrylate (PMMA) $(n=1.49)$. An array of nanofluidic channels, defined lithographically in the SU-8 film [7], constitutes the third order Bragg grating DFB laser resonator. The resonator has a phase shift of $\pi / 2$ in the middle, see Fig. 1(d), to obtain a single resonance for each Bragg reflection. The laser resonator is embedded in a $300 \mathrm{~nm}$ high $500 \mu \mathrm{m}$ wide fluidic channel and emits light laterally in the chip plane. The laser structure provides tuning of the wavelength by changing the grating period and through optofluidic functionality by changing the refractive index of the dye solution.
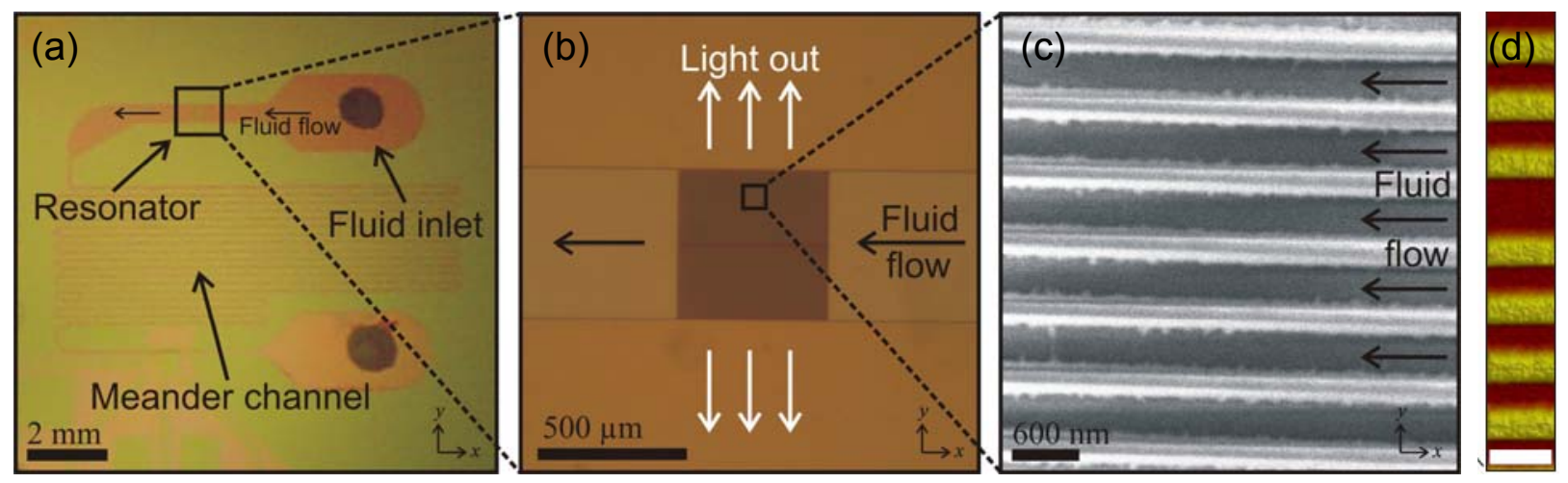

Fig. 1. Overview and close-up of the optofluidic third order DFB dye laser. (a) Image of the chip showing the fluidic network. (b) Optical micrograph of the resonator structure embedded in a microfluidic channel. (c) Scanning electron micrograph of the third order DFB laser resonator (prior to bonding). (d) Atomic force micrograph of the central region of the third order DFB laser resonator. In the middle an extra channel width is included to yield the $\pi / 2$ phase shift. The width of the white scale bar is $600 \mathrm{~nm}$. 

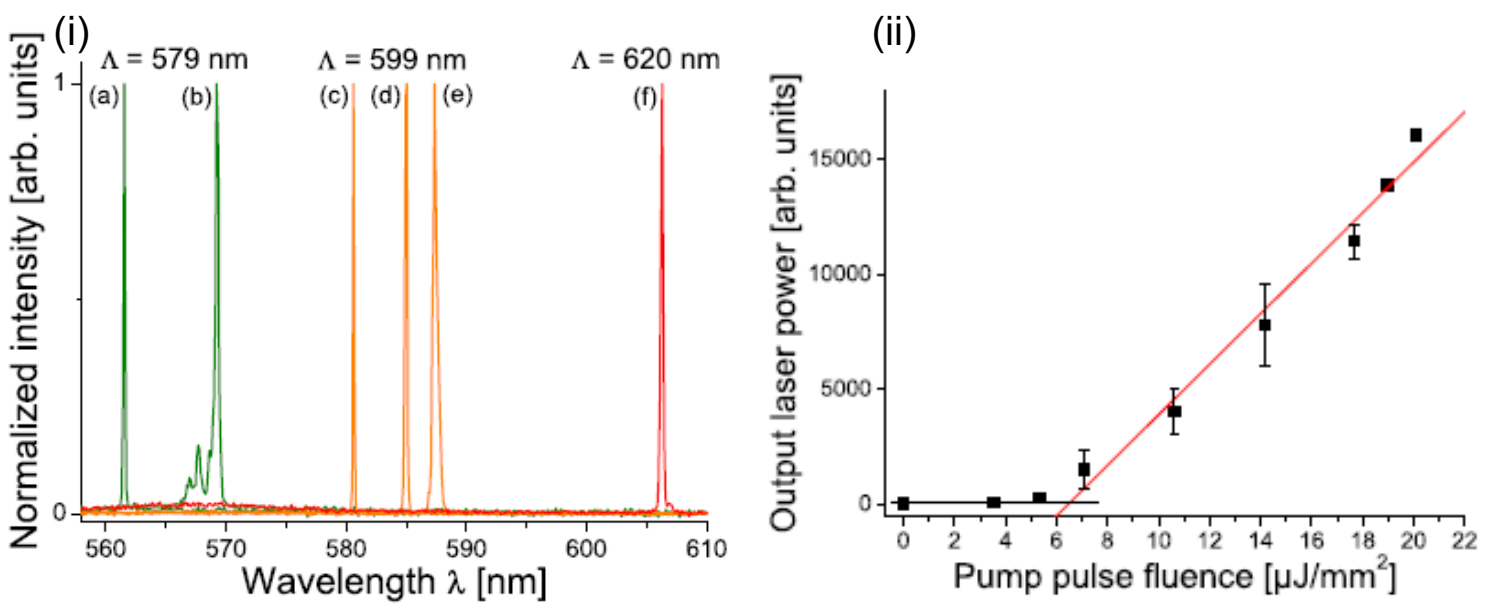

Fig. 2. (i) Laser spectra from different laser devices showing a tunability of the lasers of $45 \mathrm{~nm}$ by changing the grating period and the refractive index of the R6G solution. (ii) Typical pump pulse fluence/output laser power graph for the laser device with spectrum shown in Fig. 2(i)(e). The graph follows the standard pump/output relation of two linear segments around a laser threshold of $\sim 7 \mu \mathrm{J} / \mathrm{mm}^{2}$

To operate the devices, a droplet of liquid dye solution is applied to the fluid inlet hole and the fluidic network is filled by capillary action. Here, the laser dye rhodamine 6G (R6G) dissolved in (i) ethylene glycol ( $n=1.43)$, (ii) a 2:1 mixture of ethylene glycol and benzyl alcohol $(n=1.467)$, and (iii) a 1:1 mixture of ethylene glycol and benzyl alcohol $(n=1.485)$, all with a concentration of $2 \times 10^{-2} \mathrm{~mol} / \mathrm{L}$ is used. The laser is optically pumped at $532 \mathrm{~nm}$ perpendicularly to the chip plane by a frequency doubled Nd:YAG laser ( $5 \mathrm{~ns}$ pulse duration) and the output dye laser light is collected by an optical fiber at the edge of the chip and analyzed using a fixed grating spectrometer (resolution $0.15 \mathrm{~nm}$ ).

Lasers with three different grating periods $\Lambda=579,599,620 \mathrm{~nm}$ were fabricated by varying the width of the nanochannels and keeping the width of the SU-8 walls fixed. The periods were derived from atomic force micrographs of the SU-8 structures prior to bonding, see Fig. 1(d). The resonances of the grating satisfy the Bragg condition $m \lambda_{m}=2 \Lambda_{\text {op }}$ where $m=1,2,3, \ldots$ is the reflection order, $\lambda_{m}$ is the free space wavelength of the $m$ 'th reflection order, and $\Lambda_{\mathrm{op}}$ is the optical path length of one grating period [8]. The spatial dimensions of the resonator segments are carefully chosen so that the DFB laser oscillates at the third order Bragg reflection wavelength [6]. The low Bragg reflection order yields: (i) low out-of-plane scattering losses, (ii) low coupling losses for the light when traversing the nanofluidic channels due to the sub-wavelength dimensions of the resonator segments, and (iii) a large free spectral range $(F S R)$. Points (i)+(ii) enables a low threshold for lasing, point (iii) facilitates a wide tuning range.

Fig. 2(i) shows laser spectra for devices with different grating periods. The laser wavelength $\lambda$ is tuned by changing the grating period $\Lambda$ and the refractive index of the R6G solution. The lasers are polarized perpendicular to the chip plane (TM). From the experimental data $\Lambda_{\text {op }}$ can be estimated. Given $\Lambda_{\text {op }}$, the $F S R$ is calculated as $F S R=\lambda_{m} /(m-1)$. As an example, we obtain $\Lambda_{\mathrm{op}}=871 \mathrm{~nm}$ and correspondingly $F S R=290 \mathrm{~nm}$ for the laser with spectrum shown in Fig. 2(i)(c) (ethylene glycol solution). This very large FSR ensures that, only the third order resonance lies within the gain spectrum of the chosen dye (for visible wavelengths), thus enabling a wide tuning range. The data demonstrate a wavelength tunability of $45 \mathrm{~nm}$ by changing the grating period (coarse-tuning) and dye solution refractive index (fine-tuning).

Fig. 2(ii) shows the output laser power as a function of the average pump pulse fluence for the laser of Fig. 2(i)(e). The graph follows the standard pump/output relation of two linear segments around a threshold fluence of approximately $7 \mu \mathrm{J} / \mathrm{mm}^{2}$ and is typical for the lasers. The error bars of the output laser power reflect the pump pulse energy fluctuations from our Nd:YAG system.

\section{References}

[1] D. Psaltis, S. R. Quake, and C. Yang, "Developing optofluidic technology through the fusion of microfluidics and optics," Nature 442, 381386 (2006).

[2] E. Verpoorte, "Chip vision-optics for microchips", Lab. Chip 3, 42N-52N (2003).

[3] S. Balslev, A. M. Jorgensen, B. Bilenberg, K. B. Mogensen, D. Snakenborg, O. Geschke, J. P. Kutter, and A. Kristensen, "Lab-on-a-chip with integrated optical transducers," Lab. Chip 6, 213-217 (2006).

[4] S. Balslev and A. Kristensen, "Microfluidic Single Mode Laser Using High Order Bragg Grating and Antiguiding Segments," Opt. Express 13, 344-351 (2005). http://www.opticsinfobase.org/abstract.cfm?URI=oe-13-1-344.

[5] Z. Li, Z. Zhang, T. Emery, A. Scherer, and D. Psaltis, "Single mode optofluidic distributed feedback dye laser," Opt. Express 14, 696-701 (2006). http://www.opticsinfobase.org/abstract.cfm?URI=oe-14-2-696.

[6] M. Gersborg-Hansen and A. Kristensen, "Optofluidic third order distributed feedback dye laser," Appl. Phys. Lett. 89, 103518 (2006)

[7] M. Gersborg-Hansen, L. H. Thamdrup, A. Mironov, and A. Kristensen, "Combined electron beam and UV lithography in SU-8," submitted to Microelectron. Eng., (2006).

[8] M. Gersborg-Hansen and A. Kristensen, “Tunability of optofluidic distributed feedback dye lasers,” submitted, (2006). 